\title{
Using Human Raters to Characterize the Psychological Characteristics of GPS-based Places
}

Sandrine R. Müller University of Cambridge Cambridge, CB2 3EB, UK srm77@cam.ac.uk

\section{Gabriella M. Harari}

Stanford University

Stanford, CA 94305, USA

gharari@stanford.edu

\section{Abhinav Mehrotra}

University College London London, WC1E 6BT, UK

a.mehrotra@ucl.ac.uk

\section{Sandra Matz}

University of Cambridge

Cambridge, CB2 3EB, UK

sm917@cam.ac.uk

\section{Poruz Khambatta}

Stanford University

Stanford, CA 94305, USA

poruz@stanford.edu

\section{Mirco Musolesi}

University College London /

The Alan Turing Institute

London, WC1E 6BT, UK

m.musolesi@ucl.ac.uk

\author{
Cecilia Mascolo \\ University of Cambridge \\ Cambridge, CB3 OFD, UK \\ cm542@cam.ac.uk
}

\section{Samuel D. Gosling}

University of Melbourne /

University of Texas

Austin, TX 78712-0187, USA

gosling@psy.utexas.edu

\section{Peter J. Rentfrow}

University of Cambridge

Cambridge, CB2 3EB, UK

pjr39@cam.ac.uk

Permission to make digital or hard copies of all or part of this work for personal or classroom use is granted without fee provided that copies are not made or distributed for profit or commercial advantage and that copies bear this notice and the full citation on the first page. Copyrights for components of this work owned by others than ACM must be honored. Abstracting with credit is permitted. To copy otherwise, or republish, to post on servers or to redistribute to lists, requires prior specific permission and/or a fee. Request permissions

from Permissions@acm.org.

UbiComp/ISWC'17 Adjunct, September 11-15, 2017, Maui, HI, USA (C) 2017 Association for Computing Machinery. ACM ISBN 978-1-4503-5190-4/17/09 \$15.00

https://doi.org/10.1145/3123024.3123135

\begin{abstract}
This paper showcases an approach to combining smartphone sensing technology, web mapping services, and psychological assessments to enhance our understanding of the psychological characteristics of places. For two weeks, twenty-six students used a smartphone app that passively collected GPS sensor data. Human raters then characterized their most frequently visited places on a number of psychological characteristics, such as ambience (e.g. how safe, urban, lively a place was perceived) and personality (e.g. a place's perceived extroversion and conscientiousness). We explored the relationship between these place characteristics and participants own personality traits, showing how the personality traits of the average visitor to a location can be similar or different from the place's characteristics. We conclude with a discussion of how this approach can be used in future research on places.
\end{abstract}

\section{Author Keywords}

Mobile sensing; Mobility; GPS location; Affective place associations; Personality traits.

\section{ACM Classification Keywords}

J.4 [Computer applications]: Social and behavioral sciences---Psychology 


\section{Place characteristics}

Locations of interest, e.g. university, café, gym, shop

Subjective impressions

- Affective: Degree to which a location is e.g. clean, happy, exciting,

interesting, urban.

- Personality: Openness (artistic vs. conservative), Conscientiousness (organized vs. flexible), Extraversion (outgoing vs. reserved), Agreeableness (compassionate vs. critical), and Neuroticism (emotional vs. stable).

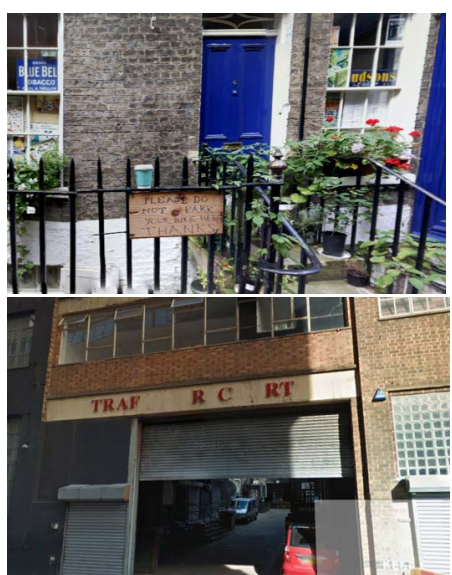

Figure 1: Example images of locations visited by users, Google Streetview data (c)2017 Google.

\section{Introduction}

Places can be characterized in terms of objective information, such as whether the place is a café, shop or park, or which socio-economic area it falls into. However, locations can also be described in terms of the psychological responses they invoke in their inhabitants. Social scientists have used people's impressions of places to understand aspects of a place, such as aesthetics, interestingness and safety (see [4, 2]). More recent technological developments, such as Google Streetview, have enabled researchers to see a $3 \mathrm{D}$ view of a location without needing to leave their office, prompting a growing body of research using such tools to understand places [5]. The aim of this paper is to showcase how smartphone sensing technology coupled with web mapping services and psychological assessments can enhance our understanding of the relationship between people's psychological characteristics and the places they choose to spend time in.

\section{Methods}

We conducted an in-the-wild investigation, using an Android app called MyLifeLogger ${ }^{1}$ to continuously track users' locations. Participants also completed validated psychological inventories assessing their personality traits.

\section{Participants}

Participants were recruited from the undergraduate population of a large UK university. 28 students downloaded the app, out of which 26 students

1 published on the Google Play Store: https://play.google.com/ store/apps/details?id=com.nsds. mystudentlife completed all study requirements and used the app for at least two weeks. The final sample consisted of 16 male and 10 female students ( $M=19.46$ years, $S D=2.18$ ), who were enrolled in 15 different undergraduate courses. $27 \%(n=7)$ of the sample was nonBritish. Participants were compensated with $£ 25$, or roughly US $\$ 30$.

\section{Significant places}

77,306 location samples were recorded across all users for the period of this study. We identified participants' significant places using existing procedures presented in [7], excluding locations where users spend less than 10 minutes overall. On average, users visited 53 significant places over the study period. The data was subsetted to contain the top ten places in which each user had spent the most time during the study period ( $M=35.25$ hours, $S D=28.44$ hours).

\section{Place characteristics}

Based on previously conducted research, we compiled a comprehensive list of coding criteria (see left) that consisted of locations of interest (based on Google Place Types, [1]) as well as affective appraisals (based on $[5,2,4])$, and personality. We used the Big Five model, which is the most widely accepted personality model [3]. The Big Five model posits five traits that reliably characterize individuals' personalities. We developed a detailed coding handbook and trained and supervised four raters, who based their ratings on Google Maps/ Streetview/Earth images of each location (see Figure 1 for example images). All locations that did not have perfect rater agreement for the locations of interest were reviewed by the first author, who is very familiar with the city the participants reside in. For 


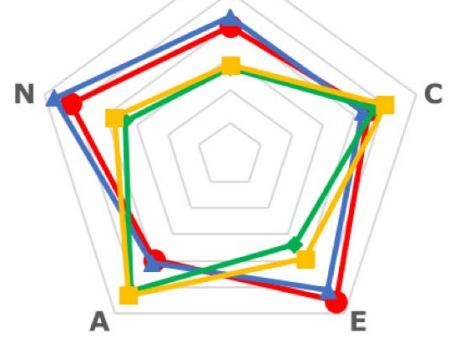

o

$\mathbf{N}$

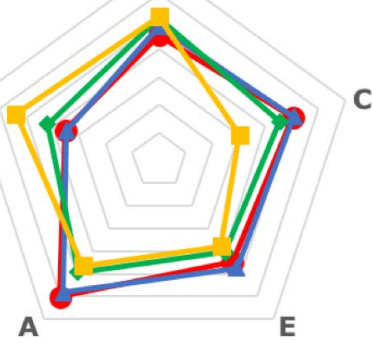

A the subjective criteria, inter-rater reliabilities were computed to evaluate rater agreement.

\section{Data Analysis}

\section{Ambience ratings}

Ambience ratings can help describe the character of a place by analyzing a specific location type's individual ambience profile. For example, streets are perceived as more lively, modern and urban compared to green spaces, while green spaces are perceived as more pleasant, relaxing, and safe (see Figure 2). Depending on the question at hand, it might also be interesting to identify places that exhibit certain characteristics (e.g. how exciting different types of locations are perceived to be by human raters; see Figure 3). Shopping malls $(M=5.37, S D=0.46)$ and cafés $(M=5.00, S D=0.25)$ are most exciting, while industrial business buildings $(\mathrm{M}=2.83, \mathrm{SD}=0.52)$ and houses/apartment buildings $(M=3.47, S D=0.68)$ are perceived to be least exciting.

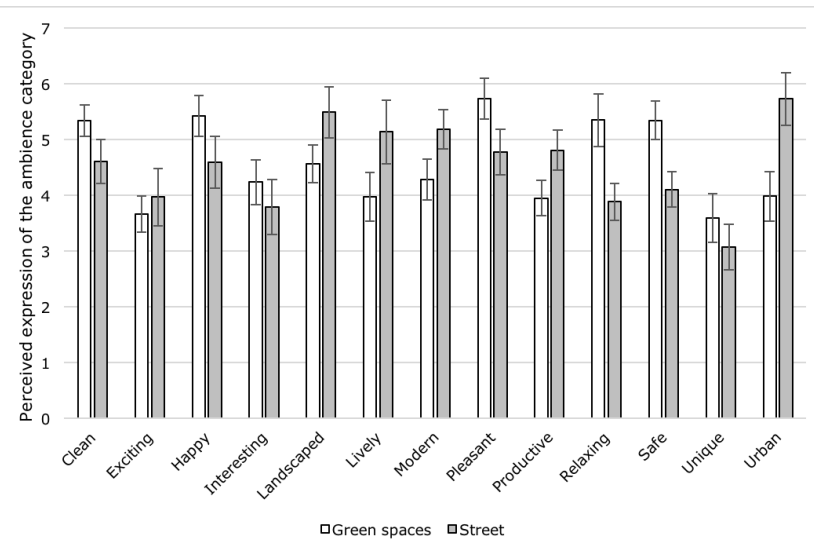

Figure 2: Ambience profiles for two different types of places.
Personality

Locations can also be analyzed with regards to their perceived personality. Figure 4 (top) shows perceiver ratings for four location categories. The perceived personality profiles of train stations and shopping malls as well as those of green spaces and religious organizations appear to be similar. For example, both green spaces and religious organizations are perceived to be highly emotionally stable and agreeable spaces, while both train stations and shopping malls are perceived to be places characterized by high extroversion and openness to new experiences.

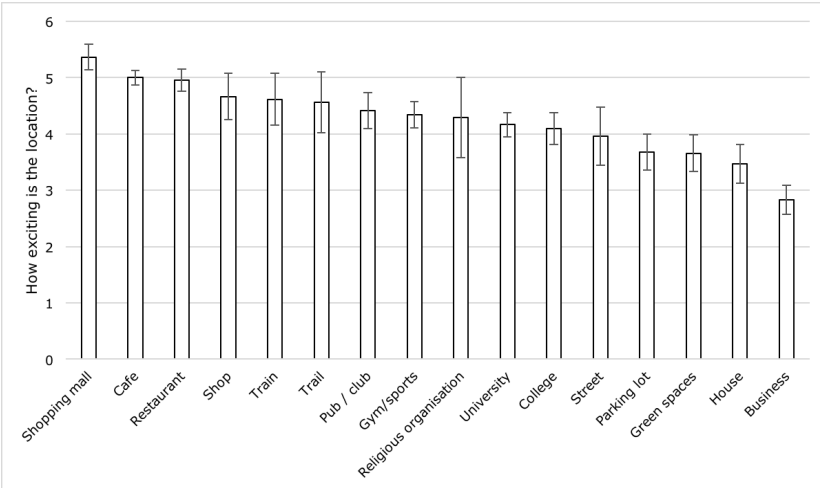

Figure 3: Ambience ratings for how exciting different types of places are perceived to be by human raters.

Interestingly, the perceived personality of a location can be compared with the actual personalities of the people that spend time there. Mapping the personality traits of the average visitor to these types of locations (weighed by time spent) yields similar patterns overall (see Figure 4, bottom). However, in this specific example, while shopping malls and train stations are similar in the types of people they attract, green spaces 
and religious organizations draw somewhat different types of individuals.

\section{Inter-rater reliabilities}

To evaluate rater agreement of the subjective coding criteria (personality and affective appraisals), interrater reliabilities were computed. A two-way inter-rater reliability agreement model was chosen as both subjects and raters are randomly chosen from a bigger pool of persons and mean rating differences between judges should be taken into account [6]. Table 1 shows the average inter-rater reliabilities across different location types. The average inter-rater reliability across all different location types is .35, which indicates low to moderate agreement between raters and is typical for measurements of similar constructs with similar amounts of raters [5]. Some location types appear to have more typical representations that result in high agreement (e.g. shopping malls .56 and cafes .53), while others might have been represented by more diverse instances in this sample (e.g. university buildings .21 or pubs/club .19). Basing analyses on larger samples than the ones presented in this investigation (both raters and sample locations rated) could also be expected to yield higher agreement. For example, previous research [e.g., 5] recommended 2232 ratings to achieve highly consistent measures.

\section{Conclusion}

The preliminary findings of this research suggest that it is possible to rate the ambience and personality of places obtained from GPS data. The approach described here can be used to understand the psychological characteristics of places and the people who spend time in them. However, more research is needed to identify approaches that improve rater reliability.

\section{Acknowledgements}

We thank the four research assistants who helped to rate the place data.

\section{References}

1. Google. 2017. Google's Place Types. Retrieved from https://developers.google.com/places

2. Kazunori Hanyu. 1997. Visual properties and affective appraisals in residential areas after dark. Journal of Environmental Psychology 17, 4: 301-315.

3. Robert R. McCrae, and Oliver P. John (1992). An introduction to the five-factor model and its applications. Journal of Personality 60, 2: 175-215.

4. Jack L. Nasar. 1998. The evaluative image of the city. Sage Publications, Thousand Oaks, CA.

5. Philip Salesses, Katja Schechtner, and César A. Hidalgo. 2013. The collaborative image of the city: mapping the inequality of urban perception. PloS One 8, 7: e68400.

6. Patrick E. Shrout, and Joseph L. Fleiss. 1979). Intraclass correlations: Uses in assessing rater reliability. Psychological Bulletin, 86, 420-428.

7. Fani Tsapeli, and Mirco Musolesi. 2015. Investigating causality in human behavior from smartphone sensor data: a quasi-experimental approach. EPJ Data Science 4, 1: 4-24. reliabilities across different location types. In brackets are the frequency with which this location type occurred across the sample. 